\title{
Ipertensione arteriosa e dilatazione dell'aorta toracica: un mistero insoluto
}

\section{Arterial hypertension and aortic root dilatation: an unsolved mystery}

\author{
Alberto Milan *, Francesco Tosello, Sara Abram, Ambra Fabbri, \\ Alessandro Vairo, Dario Leone, Franco Veglio
}

Centro per lo Studio e la Terapia dell'Ipertensione Arteriosa (Direttore: prof. Franco Veglio), SCU Medicina Interna 4, Dipartimento di Medicina e Oncologia Sperimentale, Azienda Ospedaliero-Universitaria "S. Giovanni Battista", Università degli Studi di Torino

Ricevuto l'11 febbraio 2010; accettato il 5 luglio 2010

disponibile online il 17 settembre 2010

\section{KEYWORDS \\ Arterial hypertension; Aortic root dilatation; \\ Pulse pressure; \\ Aortic dissection.}

\begin{abstract}
Summary
Introduction: Acute and chronic aortic syndromes are associated with substantial morbidity and mortality. Silent risk factors such as arterial hypertension and aortic root dilatation can increase the likelihood of aortic dissection or rupture. The relationship between arterial hypertension and the dimensions of the aortic root dimension is a topic of active debate.

Materials and methods: We reviewed the literature on the physiopathology, diagnosis, natural history, and management of thoracic aortic aneurysms.

Results: Biological variables influencing the size of the aorta include age, sex, body surface area, pressure values, and stroke volume. Pathologic enlargement of the thoracic aorta can be caused by genetic, degenerative, inflammatory, traumatic, or toxic factors. Studies investigating the correlation between aortic dimensions and arterial pressures (diastolic, systolic, or pulse) have produced discordant results.

Discussion: Classically, emphasis has been placed on the importance of hypertension-related degeneration of the medial layer of the aortic wall, which leads to dilatation of the thoracic aorta, reduced aortic wall compliance, and increased pulse pressures. However, there are no published data that demonstrate unequivocally the existence of a pathogenetic correlation between arterial hypertension and aortic root dilatation. Furthermore, there is no evidence that antihypertensive therapy is effective in the management of nonsyndromic forms of aortic root dilatation. An interesting branch of research focuses on the importance of genetic predisposition in the pathogenesis of thoracic aortic aneurysms. Different genetic backgrounds could explain
\end{abstract}

\footnotetext{
* Corrispondenza: Centro per lo Studio e la Terapia dell'Ipertensione Arteriosa, Dipartimento di Medicina e Oncologia Sperimentale, Università degli Studi di Torino, via Genova 3 - 10126 Torino.

E-mail: alberto.milan@unito.it (A. Milan).
} 
differences in the behaviour of aortic walls exposed to the same hemodynamic stress. Further study is needed to evaluate these focal physiopathological aspects.

(c) 2010 Elsevier Srl. All rights reserved.

\section{Introduzione}

Le malattie cardiovascolari sono la prima causa di morte in Occidente: nell'ambito di queste affezioni emerge la necessità di approfondire sempre più la patologia che interessi l'apparato vascolare arterioso interagente con la pompa cardiaca.

Grazie al miglioramento della capacità diagnostica e allo sviluppo e diffusione di metodiche di imaging non invasive, e in seguito al prolungamento della vita, che sottopone il cuore e l'albero arterioso a una più prolungata azione dei fattori di rischio cardiovascolare, si prende sempre più coscienza della sfida, in termini di mortalità e morbilità, rappresentata dalle sindromi aortiche acute e croniche.

\section{L'aneurisma dell'aorta ascendente}

\section{Cenni di anatomia e fisiologia}

In questo ambito risulta di particolare importanza andare a indagare la patologia aneurismatica della prima porzione dell'aorta toracica: la radice aortica sopravalvolare.

Essa è costituita, in sequenza, dall'anulus aortico, dai seni di Valsalva, dalle cuspidi e commessure della valvola aortica e dalla giunzione sino-tubulare (Fig. 1).

L'aorta toracica presenta infatti all'origine una fisiologica dilatazione, il bulbo aortico, determinata da tre piccoli rigonfiamenti, i seni aortici di Valsalva, ciascuno associato a una delle valvole semilunari dell'orifizio aortico e denominati,

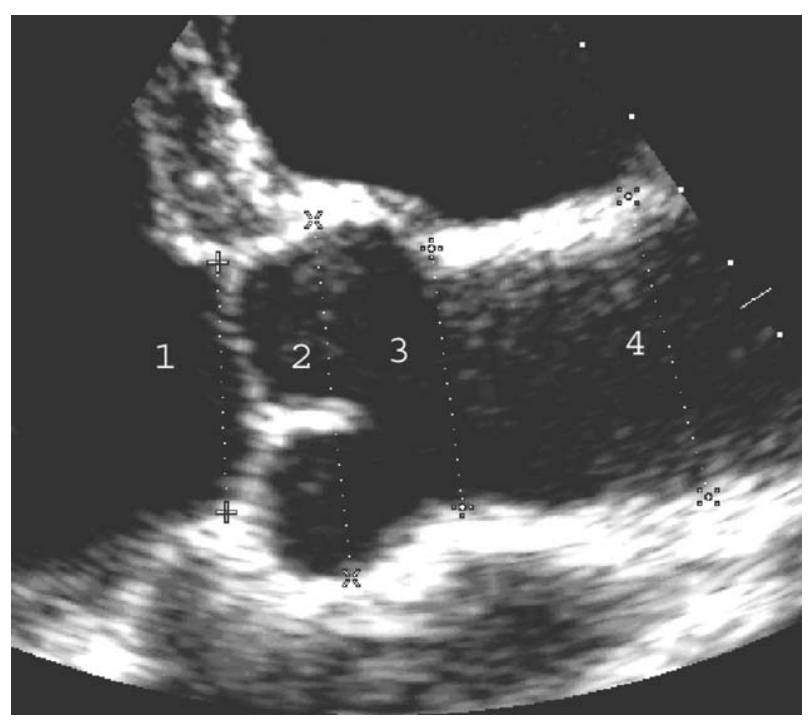

Figura 1 Visualizzazione ecocardiografica della radice aortica Legenda: 1. Anulus valvola aortica; 2. Seni di Valsalva; 3. Giunzione sino-tubulare; 4 . Aorta ascendente. rispettivamente, seno coronarico destro, seno coronarico sinistro e seno non coronarico.

L'interesse per la precisazione anatomica di questa porzione dell'aorta ha ispirato nel Rinascimento Leonardo Da Vinci, il quale cercò di valutare quale effetto emodinamico potessero esercitare i rigonfiamenti corrispondenti al bulbo aortico; in tempi più recenti la radice dell'aorta, in particolare, attirò l'attenzione del Valsalva, che nel 1740 studiò in diverse specie animali, dal punto di vista anatomico, i seni aortici che tuttora ne portano il nome, e fu sorpreso nel notare come alcune strutture e proporzioni tra di esse fossero conservate e mantenute in uccelli e mammiferi.

La radice aortica, dunque, non è solamente un breve canale di collegamento tra il ventricolo sinistro e l'aorta ascendente, ma è situata in una posizione centrale e strategica nell'ambito dell'anatomia cardiaca e vascolare, ed è strettamente correlata, anche funzionalmente, a diverse zone anatomiche fondamentali per una corretta interazione tra cuore e sistema vascolare.

Ciascuna cuspide valvolare associata alla porzione di radice aortica, al seno di Valsalva e alla cresta sovravalvolare corrispondenti crea un'unità funzionale che permette una più uniforme distribuzione del carico pressorio durante la diastole e la massima apertura delle cuspidi durante la sistole.

Queste diverse componenti modificano la loro forma e le loro dimensioni durante il ciclo cardiaco e interagiscono con le strutture circostanti per adeguarsi a condizioni emodinamiche in continua modificazione, per raggiungere l'obiettivo di minimizzare l'impedenza al flusso e il danno tissutale e di ottimizzare il flusso coronarico [1].

In caso di patologica dilatazione del normale diametro dell'aorta toracica, coinvolgente tutti gli strati della parete arteriosa e interessante uno o più segmenti di aorta toracica, si parla di aneurisma.

Non esiste una definizione quantitativa univoca della dilatazione dell'aorta toracica: le linee guida della European Association of Echocardiography (ESC) [2] indicano in $37 \mathrm{~mm}$ il cut-off per la radice aortica, mentre altri autori hanno utilizzato criteri diversi (tabella 1). L'incidenza degli aneurismi dell'aorta toracica è stimata in 6/100.000/anno, con una frequenza maggiore nella sesta e settima decade [3].

La presenza di un aneurisma aortico può inoltre essere un indice di una patologia aortica più diffusa: il $25-28 \%$ dei pazienti con aneurisma dell'aorta toracica risulta essere affetto da aneurisma aortico addominale [4].

In letteratura, il quadro anatomo-patologico che caratterizza gli aneurismi dell'aorta toracica è definito "degenerazione cistica" della media, descritto classicamente come l'associazione della perdita di cellule vascolari muscolari lisce, della frammentazione delle fibre di elastina della tonaca media della parete aortica e dell'accumulo cistico di materiale mucoide [3]. 
Tabella 1 Prevalenza della dilatazione della radice aortica in diversi studi.

\begin{tabular}{|c|c|c|c|}
\hline $\begin{array}{l}\text { Autore (anno) } \\
\text { N. pazienti [riferimento bibliografico] }\end{array}$ & Tipo di pazienti & Valore soglia/criterio & $\begin{array}{l}\text { Prevalenza } \\
\text { (\%) }\end{array}$ \\
\hline $\begin{array}{l}\text { Palmieri et al. (2001) } \\
N=2.096[13]\end{array}$ & $\begin{array}{l}\text { Popolazione generale } \\
\text { (normotesi e ipertesi) }\end{array}$ & Formula di Roman [6] & 4,2 \\
\hline $\begin{array}{l}\text { Bella et al. (2002) } \\
N=947[17]\end{array}$ & $\begin{array}{l}\text { Ipertesi essenziali con ipertrofia } \\
\text { ventricolare sinistra }\end{array}$ & Formula di Roman [6] & 10 \\
\hline $\begin{array}{l}\text { Milan et al. (2008) } \\
\text { N. } 388^{*}\end{array}$ & $\begin{array}{l}\text { Ipertesi essenziali afferenti al } \\
\text { Centro Ipertensione }\end{array}$ & $37 \mathrm{~mm}$ (maschi e femmine) & 21 \\
\hline
\end{tabular}

\section{Variabili che influenzano le dimensioni della radice aortica}

Fisiologicamente, le variabili biologiche che hanno dimostrato un'influenza sulle dimensioni della radice aortica sono l'età, il sesso, gli indici di dimensione corporea, i valori pressori e lo stroke volume.

Uno studio di Vasan et al. [5], effettuato su un'ampia coorte afferente al Framingham Heart Study, ha fornito dati desunti da un'ampia popolazione, senza patologie cardiovascolari note e priva di patologie valvolari aortiche. Questo studio ha confermato che le variabili antropometriche sono $i$ principali determinanti delle dimensioni della radice aortica: sia l'altezza sia il peso correlano linearmente con le dimensioni aortiche. $\grave{E}$ generalmente accettato che la radice aortica sia più piccola nelle donne rispetto agli uomini, ma gli studi in letteratura hanno riportato risultati contrastanti [6,7]: il modello di regressione utilizzato nello studio di Vasan et al. [5] ha indicato la presenza di una differenza media di 2,4 mm tra le dimensioni aortiche nelle donne e negli uomini, anche dopo correzione per l'età e le variabili antropometriche. Un progressivo aumento delle dimensioni aortiche con l'età è stato riportato in numerosi studi clinici e in serie autoptiche.

Un'importante quota della variabilità delle dimensioni aortiche non influenzata dall'età, dal sesso, dall'altezza, dal peso e dalla pressione arteriosa può essere attribuita all'ereditarietà: uno studio di Bella et al. [8] ha valutato, quantificandola nel $26 \%$, la proporzione della varianza delle dimensioni aortiche dovuta all'influenza genetica, dopo correzione per le variabili antropometriche e per $\mathrm{i}$ valori pressori.

\section{Pressione arteriosa e fisiologiche modificazioni delle dimensioni aortiche}

Gli studi presenti in letteratura hanno permesso di stabilire la correlazione tra le dimensioni aortiche e l'età, le variabili antropometriche e il sesso; negli ultimi anni è stata inoltre sottolineata l'importanza della predisposizione genetica nel determinismo delle dimensioni aortiche.

Per quanto riguarda la pressione arteriosa, gli studi che riportano la correlazione tra le dimensioni aortiche e i valori pressori diastolici e sistolici mostrano risultati non uniformi. Secondo un modello semplicistico, in accordo con la legge di Laplace, a una pressione di distensione maggiore dovrebbe corrispondere un maggior diametro aortico, a spese di una maggiore tensione di parete. A questo proposito Vasan et al. [5] hanno osservato, nella coorte del Framingham Heart Study, una relazione lineare tra le pressioni diastolica e media e i valori delle dimensioni aortiche: l'entità di questo effetto era minima dopo correzione per l'età e per le variabili antropometriche, ma statisticamente significativa.

Sorprendentemente, però, è stata dimostrata una relazione inversa tra i valori di pressione pulsatoria e sistolica a livello brachiale e il diametro aortico, confermata in seguito da altri lavori: lo studio di Agmon et al. [9] ha riscontrato questa relazione inversa in diversi tratti di aorta toracica. Inoltre, uno studio di Kim et al. [10] ha analizzato le dimensioni a livello della radice aortica in soggetti normotesi: le dimensioni dei vari segmenti di aorta si sono rivelate positivamente correlate solo ai valori di pressione diastolica.

\section{Ipertensione arteriosa e dimensioni aortiche}

Qualsiasi processo che indebolisca la parete dell'aorta, e la tonaca media dell'aorta in particolare, porta a un aumento dello stress parietale che può indurre la dilatazione dell'aorta e la formazione di un aneurisma, predisponendo la principale arteria dell'organismo alla dissezione e alla rottura.

È generalmente accettata la tesi che correla eziopatogeneticamente l'ipertensione arteriosa alla dilatazione della radice aortica, in quanto l'ipertensione arteriosa accelererebbe il frazionamento delle fibre di elastina nella tonaca media dell'aorta. In realtà, vari studi che hanno valutato il diametro dei seni di Valsalva telediastolico o telesistolico in relazione ai valori pressori in pazienti ipertesi sembrano non sostenere tale ipotesi.

Savage et al. [11] hanno paragonato le dimensioni della radice aortica di pazienti ipertesi e normotesi con range di età da 19 a 70 anni, e dopo correzione per età e superficie corporea (Body Surface Area, BSA) non hanno evidenziato differenze significative tra i due gruppi. Alla stessa conclusione sono giunti Virmani et al. [12], i quali hanno valutato la circonferenza aortica in un'ampia serie autoptica che includeva soggetti sia caucasici sia cinesi.

Palmieri et al. [13], in uno studio di popolazione, hanno valutato le dimensioni aortiche in pazienti ipertesi e normotesi e i soggetti affetti da dilatazione della radice aortica rappresentavano il $4,6 \%$ della popolazione considerata nel suo insieme: la prevalenza della dilatazione della radice aortica negli ipertesi è risultata del 4,2\% mentre nei normotesi del 5,8\%. In questo studio, né l'ipertensione né la durata 
dell'ipertensione risultavano correlate con le dimensioni aortiche, mentre la pressione diastolica evidenziava un debole valore predittivo indipendente per le dimensioni del diametro dei seni di Valsalva.

In un sottostudio del Systolic Hypertension in the Elderly Program (SHEP) Pearson et al. [14] non hanno rilevato differenze tra le dimensioni aortiche in soggetti affetti da ipertensione sistolica isolata e normotesi con età $>60$ anni. In un recente studio [15] è stata valutata la relazione esistente tra il diametro aortico e vari sottotipi di ipertensione in un'ampia coorte di soggetti normotesi, pre-ipertesi, affetti da ipertensione sistolica isolata, ipertensione diastolica isolata oppure ipertensione sistodiastolica. Le dimensioni aortiche nei vari gruppi rispecchiavano l'andamento correlato all'età e alle variabili antropometriche già dimostrato, ma non sono state rilevate significative differenze nel diametro aortico tra i gruppi di ipertesi e i normotesi in seguito a correzione per l'età e la BSA.

Jondeau et al. [16] hanno valutato il rapporto esistente tra il valore della pressione pulsatoria a livello centrale e il grado di dilatazione dell'aorta ascendente in pazienti affetti da sindrome di Marfan e in soggetti normali: il valore della pressione differenziale correla positivamente con le dimensioni e il diametro aortico a questo livello nei soggetti affetti; nei controlli sani, invece, i valori pulsatori centrali si sono rivelati inversamente correlati con le dimensioni aortiche.

Gli studi presenti in letteratura non sono quindi concordi nell'analisi dei rapporti tra l'ipertensione sistolica e l'associato aumento della pressione pulsatoria e le dimensioni aortiche. L'esistenza di un rapporto inverso tra diametro aortico e valori di pressione pulsatoria è sostenuta da numerosi dati in letteratura, considerando sia la pressione brachiale sia la pressione centrale [5,9,17-19].

La visione classica, che contempla un incremento consensuale dei valori emodinamici e delle dimensioni aortiche, concentra l'attenzione sull'importanza della degenerazione della tonaca media aortica secondaria all'ipertensione arteriosa, associata alla frammentazione delle fibre di elastina. Nel 1976 O'Rourke [20] ha ipotizzato un processo di "fatigue failure" relativo alle fibre di elastina. Secondo questa tesi, le fibre subiscono non solo modificazioni quantitative con l'invecchiamento, ma anche qualitative: la frammentazione e l'assottigliamento delle fibre elastiche stesse si aggiungono allo scarsa sintesi de novo di elastina. Lo scarso turnover delle fibre non compenserebbe il quantitativo di elastina che si deteriora quando soggetta per anni a ciclici carichi tensili. Inoltre, carichi pressori maggiori implicherebbero un più rapido estrinsecarsi del processo. L'ipotesi della "fatigue failure" propone una spiegazione a due delle modificazioni aortiche che più comunemente si evidenziano col trascorrere del tempo: la dilatazione e l'irrigidimento.

Questo processo porterebbe in definitiva alla dilatazione dell'aorta, con trasferimento del carico pressorio sugli elementi più rigidi della parete, le fibre collagene, e riduzione della compliance aortica. Secondo tale ipotesi si avrebbe, quindi, un aumento consensuale delle dimensioni e della rigidità aortica, associato a un incremento della velocità dell'onda pulsatoria con un più precoce ritorno dell'onda pressoria riflessa: tutti questi fenomeni esiterebbero in un incremento dei regimi pressori a livello aortico [21].

Esiste una carenza di uniformità tra le osservazioni cliniche derivate dagli studi precedentemente citati e queste previsioni teoriche: l'associazione tra incrementati valori pressori e aumento delle dimensioni aortiche non è stato sinora confermato in modo inequivocabile dalla letteratura.

\section{Ipertensione arteriosa, predisposizione genetica e dilatazione dell'aorta toracica}

Le linee guida stilate nel 2010 dell'American Heart Association (AHA) [22] per la gestione dei pazienti con ectasia dell'aorta toracica offrono un possibile spunto interpretativo, evidenziando come la variabilità interindividuale nel comportamento delle pareti aortiche in risposta agli stress emodinamici possa essere influenzata dalla differente predisposizione genetica alla dilatazione. Le linee guida enfatizzano la necessità di considerare sempre più la familiarità e la suscettibilità genetica nella valutazione della malattia aneurismatica dell'aorta toracica: fenomeni degenerativi legati all'ipertensione o all'invecchiamento influenzerebbero la patogenesi della dilatazione dell'aorta toracica in modo variabile a seconda della differente capacità predeterminata delle pareti aortiche di rispondere a queste noxae.

La predisposizione genetica si struttura in forme sindromiche $e$ in forme non sindromiche, denominate "forme familiari di dissezione e aneurisma dell'aorta toracica" [22]. Queste ultime sono forme familiari, caratterizzate da mutazioni geniche a trasmissione più frequentemente autosomica dominante: tali mutazioni sono state individuate a carico di geni codificanti per proteine strutturali (MYH11, catena pesante della beta-miosina delle cellule muscolari lisce, ACTA 2 alfa-actina delle cellule muscolari lisce) o per il recettore di tipo 2 del Transforming Growth Factor-beta (TGF-beta); queste anomalie genetiche determinano una più precoce insorgenza di aneurismi dell'aorta toracica e una più rapida progressione della dilatazione nei portatori.

Le sindromi genetiche che determinano una forte predisposizione allo sviluppo di aneurismi dell'aorta ascendente sono la sindrome di Marfan, la sindrome di Loeys-Dietz e la forma vascolare della sindrome di Ehler-Danlos.

La sindrome di Marfan (SdM) [23] è una malattia del tessuto connettivo con un'incidenza stimata di 1/5.000; la malattia è dovuta alla presenza di una delle oltre 600 mutazioni individuate a carico del gene codificante per la fibrillina-1. La SdM è una malattia autosomica dominante; nonostante ciò si pensa che almeno il $25 \%$ dei casi siano sporadici. Le manifestazioni sono pleiotropiche: scheletriche (pectus excavatum, cifoscoliosi, aracnodattilia), oculari (ectopia lentis), polmonari (bolle apicali, pneumotorace), tegumentarie e cardiovascolari (TAAD, insufficienze valvolari, prolasso mitralico). La progressiva dilatazione della radice aortica può determinare dissecazione aortica, la maggiore causa di mortalità in questo gruppo di pazienti.

Elefteriades et al. [24], in base a indicazioni desunte dall'analisi di 500 famiglie di pazienti affetti da aneurisma o dilatazione dell'aorta toracica, sono giunti ad affermare che la patologia aneurismatica di questo tratto aortico sia essenzialmente una malattia correlata ad anormalità genetiche.

Le forme sindromiche predisponenti ad aneurismi dell'aorta toracica sono divenute modello di studio per l'approfondimento della patogenesi della dilatazione dell'aorta toracica: indirettamente, gli studi includenti tali 
pazienti ipotizzano il coinvolgimento delle variabili emodinamiche nel determinare la progressione dell'ectasia aortica. Alcuni studi comprendenti pazienti con forme sindromiche di aneurisma dell'aorta toracica hanno infatti dimostrato in modo diretto la correlazione tra modificazioni degli indici emodinamici, anche centrali, e le dimensioni aortiche: Jondeau et al. [16] hanno documentato la correlazione tra $i$ valori di pressione pulsatoria centrale e la progressione della dilatazione dell'aorta toracica in pazienti affetti da SdM.

Studi di follow-up coinvolgenti pazienti con SdM hanno dimostrato indirettamente l'importanza di uno stretto controllo dei valori pressori nel ridurre la progressione della patologia aneurismatica [25].

Le stesse linee guida AHA 2010 [22], a supporto delle indicazioni alla terapia antipertensiva in tutte le categorie di pazienti affetti da dilatazione dell'aorta toracica, citano solamente studi effettuati su pazienti con SdM: sulla base dell'efficacia evidenziata in tali pazienti, le indicazioni alla terapia sono state estese anche alle forme non sindromiche di aneurismi dell'aorta toracica. In effetti le linee guida AHA 2010, pur consigliando uno stretto controllo e monitoraggio dei valori pressori in tutti i pazienti con aneurisma dell'aorta toracica, supportano tale raccomandazione di classe I con evidenze derivate da studi eseguiti in pazienti con forme sindromiche di aneurisma toracico.

\section{Interventi terapeutici}

Benché il razionale della terapia antipertensiva in pazienti affetti da ectasia dell'aorta toracica sia intuitivo, essendo teso a ridurre l'entità e il numero dei ciclici stress cui è sottoposta la parete aortica, nonché la velocità con cui si sviluppa il picco pressorio sistolico, esistono controversie a proposito della scelta dell'adeguata terapia antipertensiva.

E divenuta pratica clinica comune, in seguito alla pubblicazione dello studio randomizzato e controllato di Shores et al. [25], impostare in pazienti affetti da dilatazione dell'aorta toracica una terapia con un farmaco appartenente alla classe dei beta-bloccanti: questo studio, eseguito su 70 pazienti affetti da SdM, ha dimostrato, nel gruppo trattato con propranololo, un rallentamento significativo della progressione della dilatazione aortica.

Il benefico effetto dei farmaci beta-bloccanti in pazienti affetti da SdM è però dibattuto; inoltre, uno studio randomizzato e controllato condotto in pazienti con aneurisma dell'aorta addominale ha evidenziato un effetto paradosso della terapia con beta-bloccanti, con incremento della mortalità [26].

Le evidenze circa un possibile effetto negativo dei betabloccanti non sono però tali da sconsigliarne l'utilizzo in pazienti con ectasia localizzata a livello dell'aorta toracica: le linee guida AHA 2010 ne raccomandano l'utilizzo in classe lla in tutti i pazienti.

Inoltre studi su modelli murini hanno proposto che gli antagonisti dei recettori AT1 per l'angiotensina II (ARB) possano essere più efficaci dei beta-bloccanti nel prevenire il deterioramento strutturale della parete aortica in caso di SdM [27]. Negli ultimi anni alcune ricerche hanno messo in luce la possibilità che la fibrillina, molecola della matrice extracellulare che si ritrova in forma e quantitativo anomali nei pazienti con SdM, oltre ad avere funzioni di sostegno e connessione, sequestri e immagazzini il TGF-beta a questo livello: un ridotto quantitativo di fibrillina determinerebbe un'eccessiva attivazione della segnalazione mediata dal TGFbeta, con le conseguenti modificazioni cellulari e tissutali evidenziabili nel contesto della parete aortica di questi pazienti [28]. Gli ARB agirebbero antagonizzando l'effetto dell'aumentata espressione e attività del TGF-beta a livello delle cellule muscolari lisce: il legame dell'angiotensina II al recettore AT1 incrementa, infatti, la sintesi di TGF-beta direttamente o anche indirettamente, attraverso l'aumentata espressione dell'attivatore trombospondina 1. Il TGF-beta, a sua volta, media alcune modificazioni a carico della componente cellulare della parete aortica caratteristiche della SdM: proliferazione, fibrosi, aumentata espressione delle MMP2 e 9 (Matrix Metalloproteinase-2, Matrix Metalloproteinase-9). La somministrazione di losartan in modelli murini di SdM ha infatti dimostrato efficacia nel prevenire o attenuare le modificazioni istopatologiche a carico della parete aortica.

Brooke et al. [29] hanno presentato un lavoro in cui dimostrano che il trattamento con ARB blocca la progressione della dilatazione aortica in bambini affetti da SdM.

Le linee guida AHA 2010 hanno pertanto incluso le categorie degli ARB e degli ACE-inibitori tra le classi di farmaci indicati nel trattamento dei pazienti ipertesi con ectasia dell'aorta toracica.

\section{Conclusioni}

È generalmente accettata l'ipotesi che collega l'ipertensione arteriosa alla patogenesi dell'ectasia dell'aorta toracica; è altrettanto evidente la necessità di uno stretto controllo pressorio in tali pazienti.

La letteratura non ha sinora dimostrato con chiarezza la correlazione eziopatogenetica tra l'ipertensione arteriosa e la dilatazione dell'aorta toracica; d'altro canto non sono presenti in letteratura studi che abbiano valutato l'effettiva efficacia della terapia antipertensiva allo scopo di ridurre la progressione della patologia aneurismatica aortica in pazienti con dilatazione non sindromica. Sono inoltre stati sollevati dubbi riguardo all'effettiva sicurezza della terapia antipertensiva attuata con farmaci beta-bloccanti in pazienti con ectasia dell'aorta toracica.

Permane, in conclusione, un alone di mistero riguardo al rapporto tra l'ipertensione arteriosa e la dilatazione dell'aorta toracica sia sul versante patogenetico sia sul versante terapeutico. Una possibile chiave interpretativa è offerta dalle evidenze che in letteratura supportano la presenza di un'importante componente di suscettibilità genetica in relazione allo sviluppo e alla progressione della patologia aneurismatica dell'aorta toracica: una medesima noxa emodinamica potrebbe determinare modificazioni significativamente differenti in pazienti con pareti aortiche variabilmente predisposte dal punto di vista strutturale. La carenza di evidenze in letteratura potrebbe correlarsi proprio all'eterogeneità delle popolazioni prese in esame, determinata dalla variabilità interindividuale nella suscettibilità alla dilatazione dell'aorta toracica.

In definitiva, sono necessari ulteriori studi longitudinali per valutare quale sia la storia naturale della patologia aneurismatica dell'aorta toracica, precisare la correlazione tra valori pressori arteriosi e diametro aortico, in particolare 
tra gli indici emodinamici centrali e le dimensioni aortiche, individuare e valutare l'accuratezza di eventuali indici nel predire lo sviluppo di complicanze con alta mortalità e morbilità come la dissezione e la rottura aortica, e l'efficacia della terapia medica nel prevenirle.

\section{Conflitto di interesse}

Gli autori dichiarano di essere esenti da conflitto di interessi.

\section{Bibliografia}

[1] Vesely I. Aortic root dilation prior to valve opening explained by passive hemodynamics. J Heart Valve Dis 2000;9(1):16-20.

[2] Lang RM, Bierig M, Devereux RB, Flachskampf FA, Foster E, Pellikka PA, et al. Recommendations for chamber quantification. Eur J Echocardiogr 2006;7(2):79-108.

[3] Ince H, Nienaber CA. Etiology, pathogenesis and management of thoracic aortic aneurysm. Nat Clin Pract Cardiovasc Med 2007;4(8):418-27.

[4] Isselbacher EM. Thoracic and abdominal aortic aneurysms. Circulation 2005;111(6):816-28.

[5] Vasan RS, Larson MG, Levy D. Determinants of echocardiographic aortic root size. The Framingham Heart Study. Circulation 1995;91(3):734-40.

[6] Roman MJ, Devereux RB, Kramer-Fox R, O'Loughlin J. Twodimensional echocardiographic aortic root dimensions in normal children and adults. Am J Cardiol 1989;64(8):507-12.

[7] Kitzman DW, Scholz DG, Hagen PT, Ilstrup DM, Edwards WD. Agerelated changes in normal human hearts during the first 10 decades of life. Part II (Maturity): A quantitative anatomic study of 765 specimens from subjects 20 to 99 years old. Mayo Clin Proc 1988;63(2): 137-46.

[8] Bella JN, MacCluer JW, Roman MJ, Almasy L, North KE, Welty TK, et al. Genetic influences on aortic root size in American Indians: the Strong Heart Study. Arterioscler Thromb Vasc Biol 2002; 22(6):1008-11.

[9] Agmon Y, Khandheria BK, Meissner I, Schwartz GL, Sicks JD, Fought AJ, et al. Is aortic dilatation an atherosclerosis-related process? Clinical, laboratory, and transesophageal echocardiographic correlates of thoracic aortic dimensions in the population with implications for thoracic aortic aneurysm formation. J Am Coll Cardiol 2003;42(6):1076-83.

[10] Kim M, Roman MJ, Cavallini MC, Schwartz JE, Pickering TG, Devereux RB. Effect of hypertension on aortic root size and prevalence of aortic regurgitation. Hypertension 1996;28(1): 47-52.

[11] Savage DD, Drayer JI, Henry WL, Mathews Jr EC, Ware JH, Gardin JM, et al. Echocardiographic assessment of cardiac anatomy and function in hypertensive subjects. Circulation 1979;59(4):623-32.

[12] Virmani R, Avolio AP, Mergner WJ, Robinowitz M, Herderick EE, Cornhill JF, et al. Effect of aging on aortic morphology in populations with high and low prevalence of hypertension and atherosclerosis. Comparison between occidental and Chinese communities. Am J Pathol 1991;139(5):1119-29.

[13] Palmieri V, Bella JN, Arnett DK, Roman MJ, Oberman A, Kitzman DW, et al. Aortic root dilatation at sinuses of valsalva and aortic regurgitation in hypertensive and normotensive subjects: The Hypertension Genetic Epidemiology Network Study. Hypertension 2001;37(5):1229-35.
[14] Pearson AC, Gudipati C, Nagelhout D, Sear J, Cohen JD, Labovitz AJ. Echocardiographic evaluation of cardiac structure and function in elderly subjects with isolated systolic hypertension. J Am Coll Cardiol 1991;17(2):422-30.

[15] Farasat SM, Morrell CH, Scuteri A, Ting CT, C P Yin F, Spurgeon $\mathrm{HA}$, et al. Do hypertensive individuals have enlarged aortic root diameters? Insights from studying the various subtypes of hypertension. Am J Hypertens 2008;21(5):558-63.

[16] Jondeau G, Boutouyrie P, Lacolley P, Laloux B, Dubourg O, Bourdarias JP, et al. Central pulse pressure is a major determinant of ascending aorta dilation in Marfan syndrome. Circulation 1999;99(20):2677-81.

[17] Bella JN, Wachtell K, Boman K, Palmieri V, Papademetriou V, Gerdts E, et al. Relation of left ventricular geometry and function to aortic root dilatation in patients with systemic hypertension and left ventricular hypertrophy (the LIFE study). Am J Cardiol 2002;89(3):337-41.

[18] Farasat SM, Morrell CH, Scuteri A, Ting CT, Yin FC, Spurgeon HA, et al. Pulse pressure is inversely related to aortic root diameter implications for the pathogenesis of systolic hypertension. Hypertension 2008;51(2):196-202.

[19] Mitchell GF, Conlin PR, Dunlap ME, Lacourcière Y, Arnold JM, Ogilvie RI, et al. Aortic diameter, wall stiffness, and wave reflection in systolic hypertension. Hypertension 2008;51(1): 105-11.

[20] O'Rourke MF. Pulsatile arterial haemodynamics in hypertension. Aust N Z J Med 1976;6 suppl 2:40-8.

[21] O'Rourke MF, Nichols WW. Aortic diameter, aortic stiffness, and wave reflection increase with age and isolated systolic hypertension. Hypertension 2005;45(4):652-8.

[22] Hiratzka LF, Bakris GL, Beckman JA, Bersin RM, Carr VF, Casey Jr DE, et al. 2010 ACCF/AHA/AATS/ACR/ASA/SCA/SCAI/SIR/STS/ SVM guidelines for the diagnosis and management of patients with Thoracic Aortic Disease: a report of the American College of Cardiology Foundation/American Heart Association Task Force on Practice Guidelines, American Association for Thoracic Surgery, American College of Radiology, American Stroke Association, Society of Cardiovascular Anesthesiologists, Society for Cardiovascular Angiography and Interventions, Society of Interventional Radiology, Society of Thoracic Surgeons, and Society for Vascular Medicine. Circulation 2010;121(13):e266-369.

[23] Pyeritz RE. Marfan syndrome: current and future clinical and genetic management of cardiovascular manifestations. Semin Thorac Cardiovasc Surg 1993;5(1):11-6.

[24] Elefteriades JA, Farkas EA. Thoracic aortic aneurysm clinically pertinent controversies and uncertainties. J Am Coll Cardiol 2010;55(9):841-57.

[25] Shores J, Berger KR, Murphy EA, Pyeritz RE. Progression of aortic dilatation and the benefit of long-term beta-adrenergic blockade in Marfan's syndrome. N Engl J Med 1994;330(19):1335-41.

[26] Lindholt JS, Henneberg EW, Juul S, Fasting H. Impaired results of a randomised double blinded clinical trial of propranolol versus placebo on the expansion rate of small abdominal aortic aneurysms. Int Angiol 1999;18(1):52-7.

[27] Habashi JP, Judge DP, Holm TM, Cohn RD, Loeys BL, Cooper TK, et al. Losartan, an AT1 antagonist, prevents aortic aneurysm in a mouse model of Marfan syndrome. Science 2006;312(5770): 117-21.

[28] Kalluri R, Han Y. Targeting TGF-beta and the extracellular matrix in Marfan's syndrome. Dev Cell 2008;15(1):1-2.

[29] Brooke BS, Habashi JP, Judge DP, Patel N, Loeys B, Dietz HC 3rd. Angiotensin II blockade and aortic-root dilation in Marfan's syndrome. N Engl J Med 2008 26;358(26):2787-95. 